group $\mathrm{N}$ whose pili have only recently been seen (Bradley, Plasmid 2, 632-636, 1979). The chapter on plasmids in human and veterinary medicine gives an overview of the astonishing evolutionary phenomenon of antibiotic resistance. Very often, within a few years of the introduction of a new antibacterial drug, bacteria resistant to that drug have appeared, the resistance being plasmid-determined. The author points out (p. 138) that the molecular events leading to the appearance of new resistance plasmids may be rare, the intensity of selection for resistance making their effects quickly evident. Gentamicin resistance, for example, is already more frequent in both Gram-negative and Gram-positive bacteria than it was at the time of publication of the papers cited on pp.114-115 of this book, a result of the widespread use of gentamicin in hospitals.

The same chapter also reviews what is known of plasmids that confer pathogenicity. There is reason to hope that research on naturally occurring plasmids, and on plasmids used as cloning vehicles for 'pathogenicity' genes, will extend our present very limited understanding of the effects of bacterial infections.

A chapter entitled "Other Plasmids" describes those determining colicinogeny, degradative functions in pseudomonas, plant tumour causation by Agrobacterium tumefaciens, and antibiotic production and resistance in actinomycetes. Obviously, in such a short space there must be much abbreviation and some simplification, and indeed this applies to the whole book. The references, however, are well chosen (the method of choice is explained in the preface) and numerous enough to help the reader to find out more about any of the topics covered in the text.

I have not found many errors in this book and those I have detected are fairly trivial. (To give one example, the semisynthetic penicillins resistant to staphylococcal penicillinase are described as "cloxacillins" - they should be called isoxazolyl penicillins, of which cloxacillin is one example.) Also, considering the size of the book, I have found very few omissions. The only mention of plasmids in strictly anaerobic bacteria is that referring to Bacteroides ochraceus (p. 139); plasmids in Clostridium species as well as bacteroides might have been mentioned in Chapter 6.

Two previous single-author books on plasmids are Bacterial Plasmids by G. G. Meynell (Macmillan, 1972) and Infectious Multiple Drug Resistance by S. Falkow (Pion, 1975). Both are good books but neither, in 1980, can provide for the readers who will, I believe, gratefully welcome Broda's book.

Naomi Datta is Professor of Microbial Genetics, Royal Postgraduate Medical School, London, UK.

\section{Towards enlightenment in radioactivity}

\section{Thaddeus J. Trenn}

Radioactivity in America: Growth and Decay of a Science. By Lawrence Badash. Pp. 327. (Johns Hopkins University Press: Baltimore and London, UK, 1979.) £11.50.

THE value of history may well lie in the perspective the past can provide for the present in its relation to the future. Radioactivity is often talked around today, but generally with little understanding. Radioactive rain and snow were present long before such phenomena were discovered and investigated scientifically. Primitive man had a great fear of lightning and other forces of nature, yet with respect to radioactivity most of us still stand today as did the Neanderthals. Knowledge is power but ignorance breeds impotence. The first step to correct this source of insecurity and fear is to learn what makes it 'tick'. To this end it would be valuable to trace the historical development of man's understanding of radioactive phenomena in a manner accessible to the layman. This is what the book under review has done and why it is so important.

The science of radioactivity had a long and complex history, starting with Becquerel's discovery of natural radioactivity in 1896 and reaching to the discovery of artificial radioactivity by Joliot and Curie in 1934. A spate of major tomes, from about 1930, attests to the maturation of this multi-faceted discipline: Meyer and Schweidler, Radioaktivitdt (1927), Kohlrausch, Radioaktivität (1928), Rutherford, Chadwick and Ellis, Radiations from Radioactive Substances (1930), Curie, Radioactivité (1935), and regular reports on the subject which appeared until 1943 published in the Annual Reports on the Progress of Chemistry.

Badash narrows the subject both in time and in range by focusing upon what radioactivity research there was in America from scientists such as Boltwood, McCoy, Baskerville, Bumstead and Pegram. Rutherford and Soddy naturally play a central role not only because they were in Canada when they provided the theoretical explanation of radioactivity in 1902/3 see Nature 274, 723-724; 1978 - but also because they attracted many research workers. Indeed, part of the decline of this American scientific specialty can be attributed, as Badash suggests, to the transfer of these two scientists to the other side of the Atlantic (Chapter 18).

Much of radioactivity research in America was directed towards a clarification of the genetic connections within the several radioactive decay sequences. Such radiochemical analysis fell in between physics and traditional chemistry and could never really establish itself in America. According to Badash, this scientific specialty was also "suicidally successful"' (p. 152); it was no longer viable once the genetic connections had been sorted out in uranium, thorium and actinium. The main lines of radioactivity research thus remained in Cambridge, Paris, Rome and Vienna, leading to artificial transmutation and artificial radioactivity. Even radiochemistry took on a new life as radioactivation analysis (see Broda, Advances in Radiochemistry, Cambridge University Press; 1950). In retrospect, we can mark the transition from radioactivity to nuclear physics and nuclear chemistry with the discovery of the neutron in 1932 followed soon after by Fermi's explanation of $\beta$-decay.

Badash does not always restrict his historical account of radioactivity to the scientific specialty which had its "growth and decay" in America. When dealing with the concept of isotopy and the group displacement laws, for example, Badash leaves the American scene for two full chapters with the centre of action in Europe. $\mathrm{He}$ is overtly sympathetic with Fajan's electrochemical approach, an alternative which phenomenologically did yield nearly the complete and correct formal scheme, although it was unsound (p.211) and accordingly lacked the explanatory power of Soddy's subsequent analysis, for which he alone received the Nobel Prize.

The decline of radioactivity research in America by the end of the Great War was conditioned by factors besides the loss of Rutherford to Europe, the lack of any institutional framework and the selffulfilment of the goals of this scientific specialty. Radioactivity research "shared the same . . . poverty as other branches of science" in America (p.271). Therefore, what Badash has termed the "decay of a science" is perhaps simply the decline of a scientific sub-discipline as but one more manifestation of a general atrophy or perhaps the "immaturity" (p.274) of American science needing the fertilizing immigration of the 1930s to develop.

The reader may consider much of this somewhat remote from the current pressing concerns about nuclear reactors and atomic bombs. Nevertheless, all this began with radioactivity; the emission of radioactive radiations and the selftransmutation of one isotope to another being nuclear processes. That nuclei can also fission into larger fractions, and that under certain conditions this fission process can lead to a chain reaction are also natural phenomena that need to be better understood. Even scientists were surprised to learn recently that our technological 'eighth wonder' - the controlled fission reactor - had been anticipated by nature when dinosaurs still roamed the earth. Inherent checks and balances prevented this natural reactor from exploding like an 
atomic bomb, since the critical state, once reached, tends to turn itself off. The very heat generated by the onset of an uncontrolled chain reaction tends to liquefy the dense and compact solid substance dispersing it, perforce eliminating the concentrated condition so essential for the onset and continuity of the critical state within a mass of fissile material.

Only if a chain reaction can be achieved and executed nearly instantaneously by intentionally overcoming the barriers nature has set can the 'atomic-bomb' effect be realized. The concentration of fissionable material in a nuclear reactor is not appropriate for this effect. In so-called thermal reactors using the isotope U-235, fissile to slow neutrons (neutrons of relatively low energy which have been 'moderated' to 'thermal' velocities), the chain reaction of the nearly instantaneous type required for the 'atomic-bomb'effect simply cannot occur. For the same reason, an atomic bomb consisting solely of the rare isotope U-235 would only fizzle. It is natural uranium, U-238, which provides the main contribution to the 'atomicbomb' effect, since it can be fissioned with neutrons which are fast enough to yield a chain reaction having the required celerity. Once these and other consequences of nature's inherent control mechanisms become better known, there will be less public obsession with the alleged dangerous proliferation in the supply of U-235 and allied (slow-neutron) fissile substances like plutonium.

Yet, to have expected the present work to deal with the totality of our misunderstanding about radioactivity and its nuclear progeny would be unreasonable. The scales can be removed from the public perception only in gradual stages. That Badash has taken us a significant step forward is perhaps a sufficient achievement.

Thaddeus J. Trenn is affiliated to the MaxPlanck-Institute in Munich, FRG.

\section{Astronomical oddities}

\section{Paul Murdin}

Monsters in the Sky. By Paolo Maffei. Translated by Mirella and Riccardo Giacconi. Pp.241. (MIT: Cambridge, Massachusetts, and London, UK, 1980.) $\$ 15$.

THE monsters in the title of this book are not constellation figures, inhabitants of UFOs, dragons or other overtly mythical creatures. Monsters is used in the same sense as in the section of the Thesaurus called Unconformity, where it is associated with wonder, curiosity, missing link and queer fish. The "monsters in the sky" are thus unusual astronomical objects. They are the objects, says Paolo Maffei, which were better left out of his previous book, Beyond the Moon, on the more straightforward parts of astronomy, because they were so unusual that they were confusing. The present book assumes the elementary knowledge of astronomy contained in the previous one and builds on it to give accounts of black holes, quasars and active galaxies, the missing mass, novae and supernovae, Vulcan and transplutonian planets, and comets (a little surprisingly this, since comets are not as unusual as all that). Each chapter is written in a fair amount of detail, with the stages laid out logically by which astronomers came to their occasionally monstrous conclusions. The chapter on Eta Carinae is the most detailed in this respect, starting with its historical light curve, laying out just how Eta Carinae differs from other superficially similar kinds of stars, discussing its infrared properties and Gratton's model of the star and concluding with its possible link with the Hubble-Sandage Variables.

The book would be enjoyed by an amateur astronomer who is ready to get to grips with some detail in astronomy. The accounts of the history of the subjects with which it deals make it a book which, say, graduate students would find it profitable to read, so that their thesis on the subjects in which they may be researching might then reference more than the last couple of years' Astrophysical Journal. The bibliography to each chapter gives enough references, typically to review articles of the kind that appear in Nature, for an easy entry into the literature dating before 1976 when the first edition of the book appeared. This, incidentally, is a translation of the second Italian edition (1979). I can pay the translation the highest compliment of all, that if it was not for a few footnotes accredited to the translators, one would scarcely notice that the book was translated.

Maffei's style is engagingly informal, although always scientific. The effects of close encounters of an astronaut with the tidal forces and event horizon of a black hole, for instance, are described with enough realism to make the image specific but not overplayed in the repellently sensational, even mystical, way which characterizes many journalistic accounts. On the contrary, this is a book which is written by an astronomer who knows the astronomical literature and has considerable skill in interpreting it at a popular level.

Paul Murdin is an astronomer at the Royal Greenwich Observatory, Herstmonceux, UK.

\section{The AI saga}

\section{Donald Michie}

Machines Who Think. By P. McCorduck. Pp.375. (Freeman: Oxford, 1979.) $\mathfrak{1 7 . 8 0}$.

THE task of the scientific referee is a well known contradiction. If he is himself a part of that particular action, then he cannot be impartial. If he is not, then how can he be technically competent in his task? The writing of scientific history is in similar case. It is therefore the more remarkable that Pamela McCorduck's gossipy saga on artificial intelligence, aspiring neither to impartiality nor to technical competence, has scored an undoubted overall success.

Her partiality is to the American part of the story, especially to Pittsburgh. Her saving gift is vitality, which carries her eagerly into previously unexplored catacombs of AI's pre-history. Using gold as the construction material the lame Greek god Hephaistos fashioned female attendants to help him walk. These robots not only walked, but also gave utterance from the promptings of inbuilt intelligence. A bronze android named Talos, Zeus' love-gift to Europa, patrolled the shores of Crete three times a day and hurled rocks at invaders. On catching any, he would heat himself up and fry the victim in a fiery embrace. Pandora, another female android from Hephaistos' shop, was developed under contract from Zeus to punish mankind for accepting Prometheus' gift of fire.

Joseph Golem was the artefact of the 16th century Loew, chief rabbi of Prague and friend of Johannes Kepler. The Golem's use was to spy on Gentiles. In reporting on his missions he was somewhat hampered by being mute.

Mary Shelley's tale is brought hauntingly to life, with Dr Frankenstein's creation driven half-mad with loneliness and his maker's evident physical repugnance for him. "Misery makes me a fiend!" says the 8-ft monster, and begs his master to fashion a soul-mate for him. Frankenstein, having promised this, abandons the task, with devastating effect on the creature's subsequent behaviour.

McCorduck's shrewdest move is to have gathered lengthy oral histories from numerous tape-recorded interviews for later interweaving with pre-existing records. It is startling to read Marvin Minsky's words of 1956:

The important result that would be looked for would be that the machine would tend to build up within itself an abstract model of the environment in which it is placed. If it were given a problem, it could first explore solutions within the internal abstract model of the environment and then attempt external experiments. Because of this preliminary internal study, these external experiments would appear to be rather clever, and the behaviour would have to be regarded as rather 'imaginative'. 